Service social

\title{
Guide méthodologique pour la recherche et l'action sociale, par André Jacolo, Montréal, Nouvelle frontière, 1984, 65 pages.
}

\section{René Auclair}

Volume 34, numéro 2-3, 1985

L’organisation communautaire

URI : https://id.erudit.org/iderudit/706285ar

DOI : https://doi.org/10.7202/706285ar

Aller au sommaire du numéro

Éditeur(s)

École de service social de l'Université Laval

ISSN

1708-1734 (numérique)

Découvrir la revue

Citer ce compte rendu

Auclair, R. (1985). Compte rendu de [Guide méthodologique pour la recherche et l'action sociale, par André Jacob, Montréal, Nouvelle frontière, 1984, 65 pages.] Service social, 34(2-3), 411-412. https://doi.org/10.7202/706285ar d'utilisation que vous pouvez consulter en ligne.

https://apropos.erudit.org/fr/usagers/politique-dutilisation/ 
Avec cet ouvrage, tout n'est pas dit sur les pratiques de conscientisation au Québec, au contraire. Ce que les auteurs nous communiquent, c'est l'état de leur cheminement au moment où le livre a été écrit.

Je termine en reprenant leurs paroles lors du lancement, à Québec : "Qui va écrire le prochain livre sur les pratiques de conscientisation au Québec?". J'espère que nous n'aurons pas trop longtemps à attendre.

\section{C.L.S.C. Sud-Ouest-Centre,}

Ronald DUHAIME Sherbrooke.

Guide méthodologique pour la recherche et l'action sociale, par André JACOB, Montréal, Nouvelle frontière, 1984, 65 pages.

Ce livre se situe d'emblée dans la catégorie des guides portant sur la méthodologie du travail intellectuel. Il vise en particulier les trois objectifs suivants: fournir les règles de base pour la recherche académique; renseigner sur les techniques de recherche et des sources d'information; outiller les étudiants et les étudiantes pour la réalisation de leurs travaux.

Cet ouvrage est divisé en sept parties. Dans la première, l'auteur distingue la recherche de la pratique sociale. Les suivantes traitent des critères pour une recherche progressiste; des types de recherche-action à privilégier; des premiers pas vers la recherche; comment réaliser la recherche; une conclusion générale; et une bibliographie, utile pour la recherche.

La recherche-action est habituellement entreprise dans le but de transformer une situation ou dans celui, plus modeste, d'énoncer des recommandations qui, mises en ouvre, changeraient cette situation. Selon que son but immédiat est de susciter des changements (par des processus aussi divers que l'innovation, l'animation sociale, le développement communautaire) ou d'étudier la situation pour éventuellement la transformer, les méthodologies peuvent varier considérablement (recherche, fonction mixte, animation sociale).

Rappelons que les options en recherche-action peuvent être résumées de la façon suivante: l'approche mécaniste; l'approche techno-systémique; l'approche organique; l'approche autogérée. Rappelons également que ces approches ne sont pas des étapes mais des visions particulières de la rechercheaction. C'est pourquoi nous ne pouvons comparer des conceptions différentes qui ne peuvent être placées sur une même ligne.

Dans le cas présent, l'auteur a choisi l'approche autogérée, qui se fonde sur les vues marxistes de la société. Les théoriciens du socialisme (Marx, Fourier, Proudhon) ont montré comment le mouvement ouvrier révélait, par ses luttes, les failles du système capitaliste. Le mouvement devenait alors l'analyseur du capitalisme industriel du 19 e siècle. D'autre part, l'aspiration profonde du socialisme se concrétisait dans une société autogérée qui devait suivre, en principe, la destruction de l'appareil d'état bureaucratique. 
Le paradigme de la recherche-action autogérée poursuit essentiellement les fins suivantes:

- proposer une conception des institutions en termes de régulation par la base, de critique permanente des normes instituées, de développement de forces instituantes ;

- démasquer le complot des institutions qui consiste à imposer, par la contrainte matérielle (économique ou physique) et idéologique, une vision fausse des rapports de production;

- montrer comment l'État, l'économie, la lutte des classes, traversent les institutions ;

- remettre à leur juste place le niveau du groupe et le niveau de l'organisation ; la psychologie des groupes et la sociologie des organisations n'expliquent pas toute la réalité.

En résumé, l'auteur a davantage mis l'accent sur les techniques et les premiers pas vers la recherche que sur une présentation systématique de l'approche autogérée de la recherche-action (fonction épistémologique, fonction culturelle, fonction politique, fonction sociale).

Ce guide s'adresse aux étudiants, aux travailleurs sociaux et à tous ceux qui, dans leur engagement social, ont besoin d'outils pour organiser une action collective.

École de service social,

René AUCLAIR

Université Laval.

Les groupes populaires à Sherbrooke : pratique, financement et structure, par Jean-Pierre Deslauriers et Hermance POULIOT, Département de service social, Université de Sherbrooke, 1982, 91 pages.

Enfin une recherche sociale qui se lit comme un roman! Et quel roman ! Je l'ai savouré comme des bretzels. Imaginez un thème de recherche qui met en vedette les groupes populaires dans une mise en scène d'inspiration "qualitative" dont le scénario met en jeu acteurs et auteurs cherchant avec une complicité sans détour à reconstituer intuitivement le réel.

Je l'aurais moi-même honni dans un passé récent alors que je vénérais le syllogisme en barbara, la méthode expérimentale et la pyramide bureaucratique de Max Weber. Après avoir constaté, d'une part, que le discours et la pratique sociale ne répondent pas au savoir-faire quantitatif comme le font les sciences de la nature, et m'être converti, d'autre part, à l'idée que des stimuli au niveau des forces sociales microcosmiques peuvent engendrer des changements sociaux, j'ai changé mon fusil d'épaule. Je crois que, pour percevoir des changements dans toute leur plénitude, il faut recourir à la méthode d'analyse qualitative et à ses procédés empreints "d'attitudes égalitaires".

L'histoire se répète disent certains. À lire Deslauriers et Pouliot, on se croirait plongé au milieu des chercheurs de l'École de Chicago des années 1920 